\title{
Experimental Observation of Oscillating Wave Propagation on Switch Lines for Generation of Continuous Electromagnetic Waves
}

\author{
Koichi Narahara and Akihiro Yokota \\ Graduate School of Science and Engineering, Yamagata University, 4-3-16 Jonan, Yonezawa, Yamagata 992-8510, Japan \\ Correspondence should be addressed to Koichi Narahara, narahara@yz.yamagata-u.ac.jp \\ Received 25 September 2008; Accepted 30 November 2008 \\ Recommended by Michel Nakhla
}

We report the experimental observation of the generation of continuous electrical waves in a switch line, which is a transmission line periodically loaded with electronic switches. The oscillating motions of a wave front have been experimentally demonstrated in a line with discrete Esaki diodes employed as switches, when a rising step-pulse signal was passed through the line.

Copyright (C) 2009 K. Narahara and A. Yokota. This is an open access article distributed under the Creative Commons Attribution License, which permits unrestricted use, distribution, and reproduction in any medium, provided the original work is properly cited.

A switch line $[1,2]$, defined as a lumped transmission line containing a series resistor, a shunt capacitor, and a shunt switch (switch open for voltages greater than a fixed threshold; switch closed otherwise) in each section, is first discussed by Richer in 1966 . We investigated a switch line for use in high-speed electronics, and found an interesting propagation property of the wave front observed when a step pulse is input: the wave front travels forward at the beginning, and then returns to the input port [3]. The returned wave is reflected at the input port; therefore this oscillating behavior continues indefinitely. By extracting a part of these waves from a switch line, we can generate an oscillatory electromagnetic wave. The oscillation frequency is determined by the round-trip time of the traveling wave; thus, it can be increased by decreasing the traveling distance or increasing the wave velocity, as long as it is not limited by the cut-off value of the operation frequency of the loaded switches.

Figure 1(a) shows two sections of a switch line, where $L$ and $R$ are the series inductor and series resistor of the unit section, respectively. A shunt switch is modeled by a capacitor $C$ and a bias-dependent conductance is placed in parallel. The ideal current-voltage relationship is shown by the solid curve in Figure 1(b). We can then write the current through the switch as $G V \theta\left(V_{\text {th }}-V\right)$, where $\theta(V)$ shows the Heaviside function $(\theta(x)=1$ for $x>0 ; \theta(x)=0$ otherwise). The current-voltage relationship of the typical tunneling diodes is shown by the dotted curve in Figure 1(b). If the voltage range that exhibits the negative differential resistance becomes narrow and if the thermal currents at the greater voltages than the valley are negligible, the tunneling diodes such as the Esaki diodes and the resonant tunneling diodes (RTDs) can be good candidates for the switches. We define two dimensionless variables $\alpha$ and $\beta$ for convenience:

$$
\begin{aligned}
& \alpha=\frac{R}{2} \sqrt{\frac{C}{L}}, \\
& \beta=\frac{G}{2} \sqrt{\frac{L}{C}} .
\end{aligned}
$$

Hereafter, we call the voltage range greater (less) than $V_{\text {th }}$ region I (II). Then, the voltage wave is influenced by the finite shunt conductance in region II and not by the conductance in region I. We consider the situation when the input rising step-pulse crosses $V_{\text {th }}$ as shown in Figure 1(c). When the wave front travels forward, it is expected that an exponential wave develops in region II, while the ordinary sinusoidal wave develops in region I. By examining the dispersion relationship, the exponential mode in region II can travel forward, when

$$
\alpha \beta>\sinh ^{2} \frac{k}{2}
$$




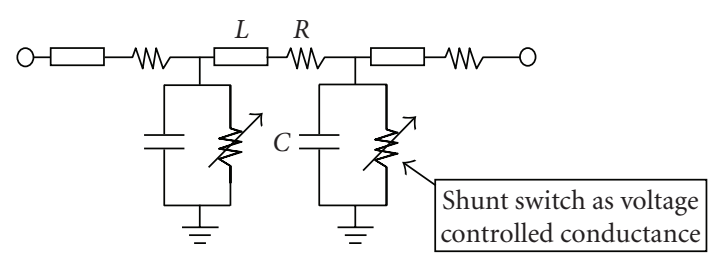

(a)

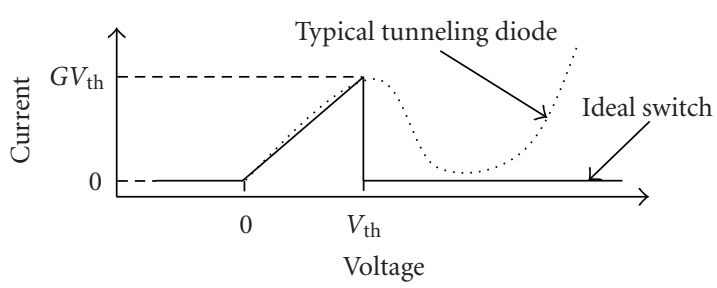

(b)

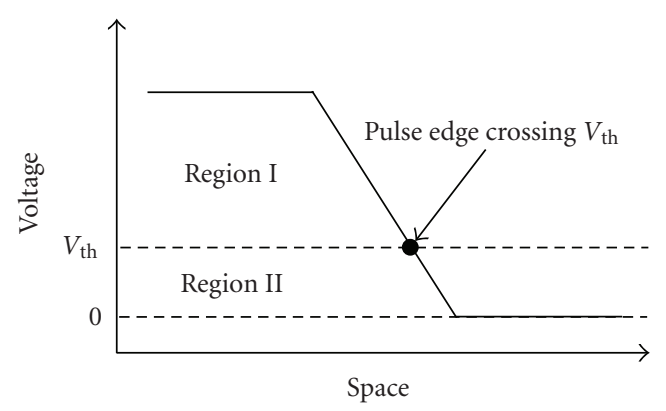

(c)

Figure 1: Definitions. (a) Equivalent representation of switch line. $L$ : series inductance, $R$ : series resistance, $C$ : shunt capacitance, $G$ : shunt conductance simulating loaded switch; (b) current-voltage relationship of shunt switch; (c) pulse input to the line (the voltage range greater (less) than $V_{\text {th }}$ is called region I (II)).

where $k$ represents its wave number normalized by the inverse of the length of the unit cell. On the other hand, the sinusoidal mode in region I has to satisfy

$$
\alpha<2 \sin \frac{k}{2} .
$$

By combining (2) and (3), we obtain the necessary condition of the development of the sinusoidal-exponential wave:

$$
\beta>\beta_{0} \equiv \frac{1}{\alpha} \sinh ^{2}\left[\sin ^{-1}\left(\frac{\alpha}{2}\right)\right] .
$$

This exotic pulse is not stable; therefore it becomes attenuated with transmission and finally disappears. At this point, a stable dynamical-steady-state (dss) pulse develops at the point where the forward pulse disappears, and starts to travel backward [4]. This process is illustrated in Figure 2. The waveforms in Figure 2 result from a time-domain finitedifference calculation of a switch line with ideal electronic switch. The spatial position on the line is shown on the horizontal axis, and the voltage is shown on the vertical axis. Figure 2(a) shows the behavior of the forward pulse. The forward pulse combining a sinusoidal mode in region I and

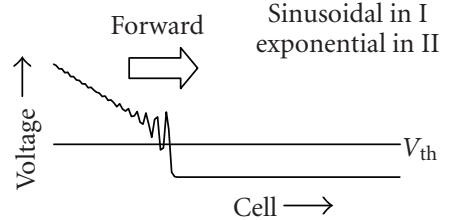

(a)

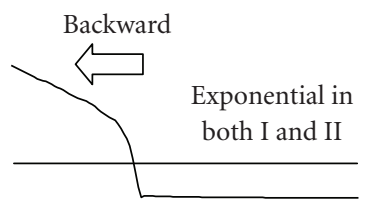

(c)

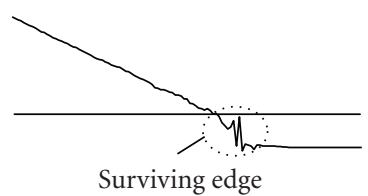

(b)

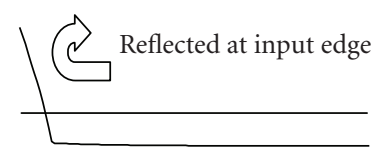

(d)
FIGURE 2: Oscillating behavior of a pulse traveling on a switch line. (a) Forward-going quasisteady pulse; (b) pulse at the turning point; (c) backward-going dss pulse; (d) reflection at input port.

an exponential mode in region II is not stable; therefore it becomes attenuated and finally disappears, so that only the exponential pulse edge is left in region II, as shown in Figure 2(b). This edge develops an exponential mode in region I to form a dss pulse, and then it starts to travel backward as shown in Figure 2(c). When the backward dss pulse reaches the input end, it is reflected as shown in Figure 2(d), so that it again starts to travel forward as an unstable sinusoidal-exponential wave. The cycle continues indefinitely with suitable boundary conditions at the input port (the input requires the lower-impedance termination for time-invariant voltage supply), thus succeeding in the generation of continuous electromagnetic waves.

Because the increase in $R$ contributes to the decrease in the round-trip distance, the oscillation frequency becomes higher for larger $R$. The increase in $G$ has the same effect. Moreover, the larger part of the pulse edge lies in region II for larger $V_{\text {th }}$. Therefore, the increase in $V_{\text {th }}$ may also decrease the round-trip distance. On the other hand, when the amplitude of the input pulse is increased, the round trip time increased; as a result, the oscillation frequency, defined as the inverse of the period of the oscillating pulse edge, decreases. This simple relationship allows the oscillation frequency to be widely tuned by varying the voltage amplitude of the input pulse. In contrast to the conventional oscillator, the device does not include any LC tanks for resonance that limit the oscillation bandwidth. By proper design of loss elements such as electrode loss and switch conductance and/or signal application, we can obtain an oscillator having the required oscillation frequency with rather a wide tuning range.

Although we have to allow for the presence of finite conductance in regions I and II, the shunt electronic switches can be designed using the devices that exhibit negative differential resistance, such as Esaki diodes or RTDs $[5,6]$, the peak voltages of which function as $V_{\text {th }}$ of the switches. Although the above discussion is based on ideal switch lines, the same properties have been established for the switch lines implemented with these tunneling devices through SPICEand finite-difference-time-domain-based calculations [7]. 
In the experiments, we used a switch line whose unit section is represented by Figure 1(a). Thirty-six sections were devised. The circuit was built on a standard bread board. The shunt electronic switches were constructed with NEC 1S1763 Esaki diodes. The peak current and voltage, the latter of which corresponds to $V_{\text {th }}$ in the ideal switch model, are typically $6.0 \mathrm{~mA}$ and $60 \mathrm{mV}$, respectively. Moreover, the typical parasitic capacitance is $30.0 \mathrm{pF}$. Series inductances, resistances, and shunt capacitances were implemented using $1.0 \mu \mathrm{H}$ inductors (TDK SP0508), $1.0 \Omega$ resistors (Tyco Electronics CFR25J), and $470 \mathrm{pF}$ capacitors (TDK FK18C0G1), respectively. The size of the unit cell is about $10 \mathrm{~mm}$. The design parameters using these components are estimated as $L=1.0 \mu \mathrm{H}, C=500 \mathrm{pF}, R=1 \Omega(\alpha=0.02)$, and $G=$ $0.1 \mathrm{~S}(\beta=4.47)$. Because the condition $\beta>\beta_{0}$ is satisfied for the present parameters, the oscillating motion of the wave front is expected. The test switch line was fed with the signal generated by an Agilent 81150A function generator. The signals along the test switch line were detected and monitored in the time domain using an Agilent DSO90254A oscilloscope. In order to monitor the waveforms using the oscilloscope, we input a pulse having finite duration instead of a step pulse. The input pulse had rise and fall times of $1.0 \mu \mathrm{s}$ with a duration of $30.0 \mu \mathrm{s}$. The output impedance of the function generator was set to $50 \Omega$.

Figure 3 shows voltage waveforms monitored at the first cell. Figure 3(a) shows a measured waveform resulting from a single sweep with the oscilloscope. Although the input signal was a simple pulse, we can observe a cycle of shortperiod pulses. Because of the weakness of the coherence between the input step pulse and the short-period pulses, the temporal positions of short-period pulses varied for different single sweeps. We numerically solved the following transmission equation of the test switch line by the standard finite difference method [8]:

$$
\begin{gathered}
L \frac{d I_{n}}{d t}=-R I_{n}+V_{n}-V_{n+1}, \\
C \frac{d V_{n}}{d t}=-I_{\text {dio }}\left(V_{n}\right)+I_{n-1}-I_{n},
\end{gathered}
$$

where $I_{n}$ and $V_{n}$ are the current and voltage at the $n$th cell. The Esaki diodes are modeled by the voltage-controlled current source $I_{\text {dio }}$, whose current-voltage relationship is given by the data sheet of 1 S1763. The variation of parameters of each device is neglected. The resulting waveform at the first cell is shown in Figure 3(b). Although the calculation does not simulate the amplitude and period exactly, it qualitatively characterizes the measured short-period pulses. By examining the numerically obtained waveforms at various cells, we see that the generation of short-period pulses results from the oscillating propagation of the edge of the input step pulse. The measured waveform of the short-period pulse is typically shown in the inset of Figure 3(a). It exhibits a trapezoid-like shape with oscillation. When the pulse edge passes a point, the voltage at the point remains constant until the edge returns. Moreover, the forward edge is carried by the sinusoidal-exponential hybrid mode. These observations are consistent with the measured pulse shape.

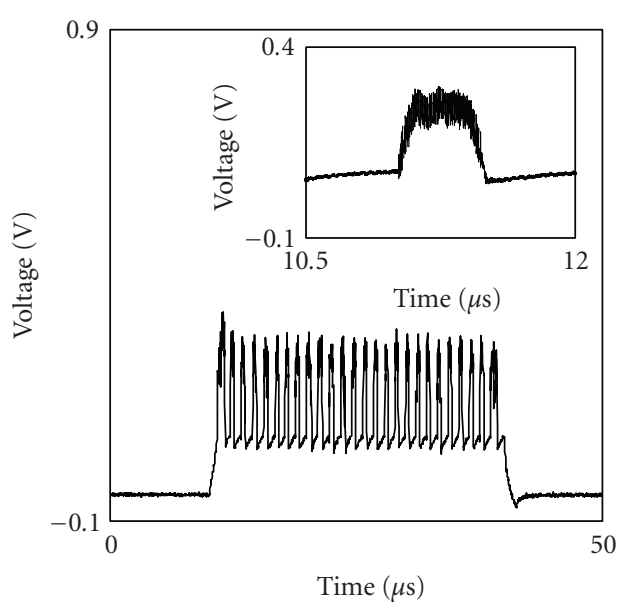

(a)

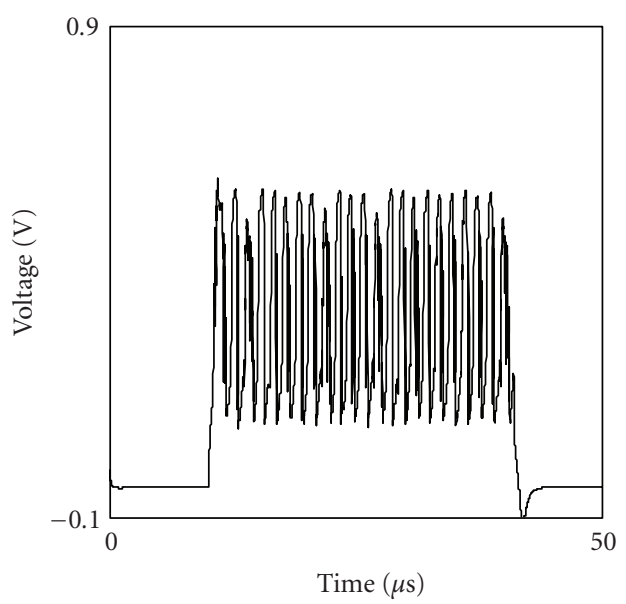

(b)

FIgURE 3: Measured waveforms monitored at first cell. (a) Waveform measured using a single sweep with the oscilloscope (the inset shows the typical waveform of the short-period pulses); (b) the waveform calculated by numerically solving the transmission equations of the test switch line.

Figure 4(a) shows the measured waveform monitored at the first cell, which was averaged 1024 times with the oscilloscope. As mentioned above, the short-period pulses have weak coherence with the step input. Through the waveform averaging, we can extract the coherent part. It is interesting to note that the averaged amplitude of the short-period pulse becomes smaller, that is, the degree of decoherence becomes greater, with time after the rising edge of the input step pulse. By using the coherent parts, it is possible to compare the phase of the voltage waveform at a cell with that at the other cells. The spatiotemporal distribution of the averaged voltage wave is shown in Figure 4(b). The waveforms at the first twenty cells having a $7.5 \mu$ s duration are plotted. The edge first travels forward. Then, at the turning point $P_{i}(i=1,2,3,4)$ in Figure $4(\mathrm{~b})$, the wave edge starts to travel backward. The backward wave front is reflected at the input port, starts to travel forward again, and reaches the turning point $P_{i+1}$. This spatiotemporal 


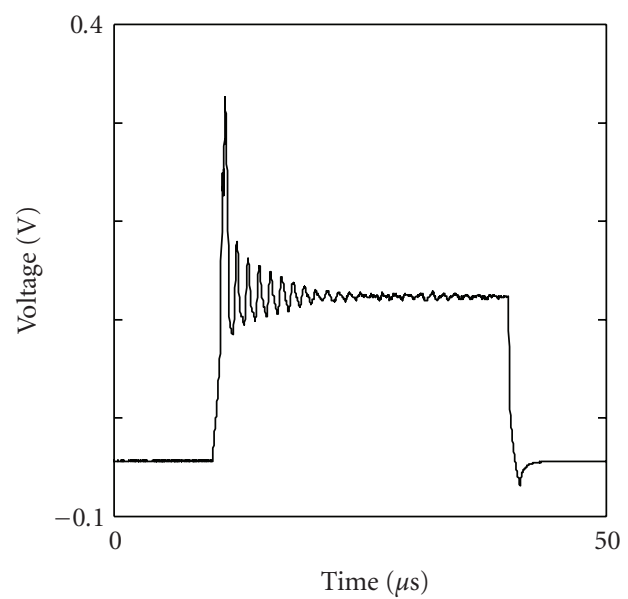

(a)

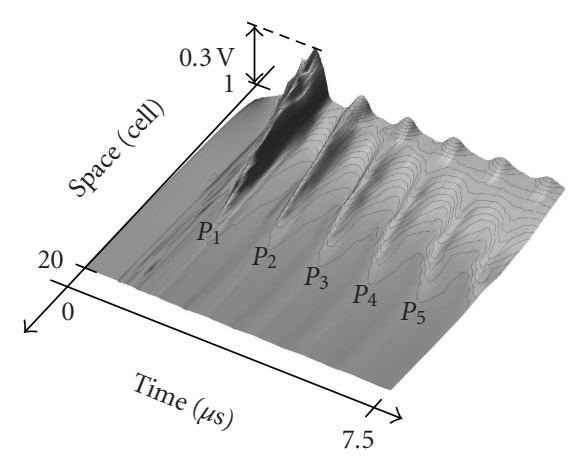

(b)

Figure 4: Coherent part of measured waveforms. (a) The waveform measured by averaging 1024 times with the oscilloscope; (b) spatiotemporal voltage distribution. Waveforms measured at the first twenty cells with a duration of $7.5 \mu$ s are plotted.

voltage distribution clearly shows that the oscillating motion of the edge is established in the test switch line.

In conclusion, we successfully demonstrated the generation of electrical continuous waves using switch lines. We believe that further investigations result in the quantified characterization of the oscillating pulse edge, such that we can manage this oscillating phenomena as we need. Our approach could be also scaled from its current $\mathrm{MHz}$ form into microwave, millimeter-wave, and terahertz form, when implemented with the state-of-the-art tunneling devices such as InP RTDs.

\section{References}

[1] I. Richer, "The switch-line: a simple lumped transmission line that can support unattenuated propagation," IEEE Transactions on Circuit Theory, vol. 13, no. 4, pp. 388-392, 1966.

[2] A. Scott, Active and Nonlinear Wave Propagation in Electronics, Wiley-Interscience, New York, NY, USA, 1970.

[3] K. Narahara, "Electromagnetic continuous-wave generation using switch lines," Journal of Applied Physics, vol. 100, no. 6, Article ID 064908, 5 pages, 2006.

[4] K. Narahara, T. Yamaki, T. Takahashi, and T. Nakamichi, "Characterization of voltage-controlled oscillator using RTD transmission line," International Journal of High Speed Electronics and Systems, vol. 17, no. 3, pp. 577-584, 2007.

[5] H. Sugiyama, H. Matsuzaki, Y. Oda, H. Yokoyama, T. Enoki, and T. Kobayashi, "Metal-organic vapor-phase epitaxy growth of InP-based resonant tunneling diodes with a strained $\mathrm{In}_{0.8} \mathrm{Ga}_{0.2}$ As well and AlAs barriers," Japanese Journal of Applied Physics, Part 1, vol. 44, no. 10, pp. 7314-7318, 2005.

[6] N. Orihashi, S. Hattori, S. Suzuki, and M. Asada, "Experimental and theoretical characteristics of sub-terahertz and terahertz oscillations of resonant tunneling diodes integrated with slot antennas," Japanese Journal of Applied Physics, Part 1, vol. 44, no. 11, pp. 7809-7815, 2005.

[7] K. Narahara and A. Yokota, "Full-wave analysis of quasi-steady propagation along transmission lines periodically loaded with resonant tunneling diodes," Japanese Journal of Applied Physics, Part 1, vol. 47, no. 2, pp. 1126-1129, 2008.

[8] C. R. Paul, Analysis of Multiconductor Transmission Lines, WileyInterscience, New York, NY, USA, 1994. 

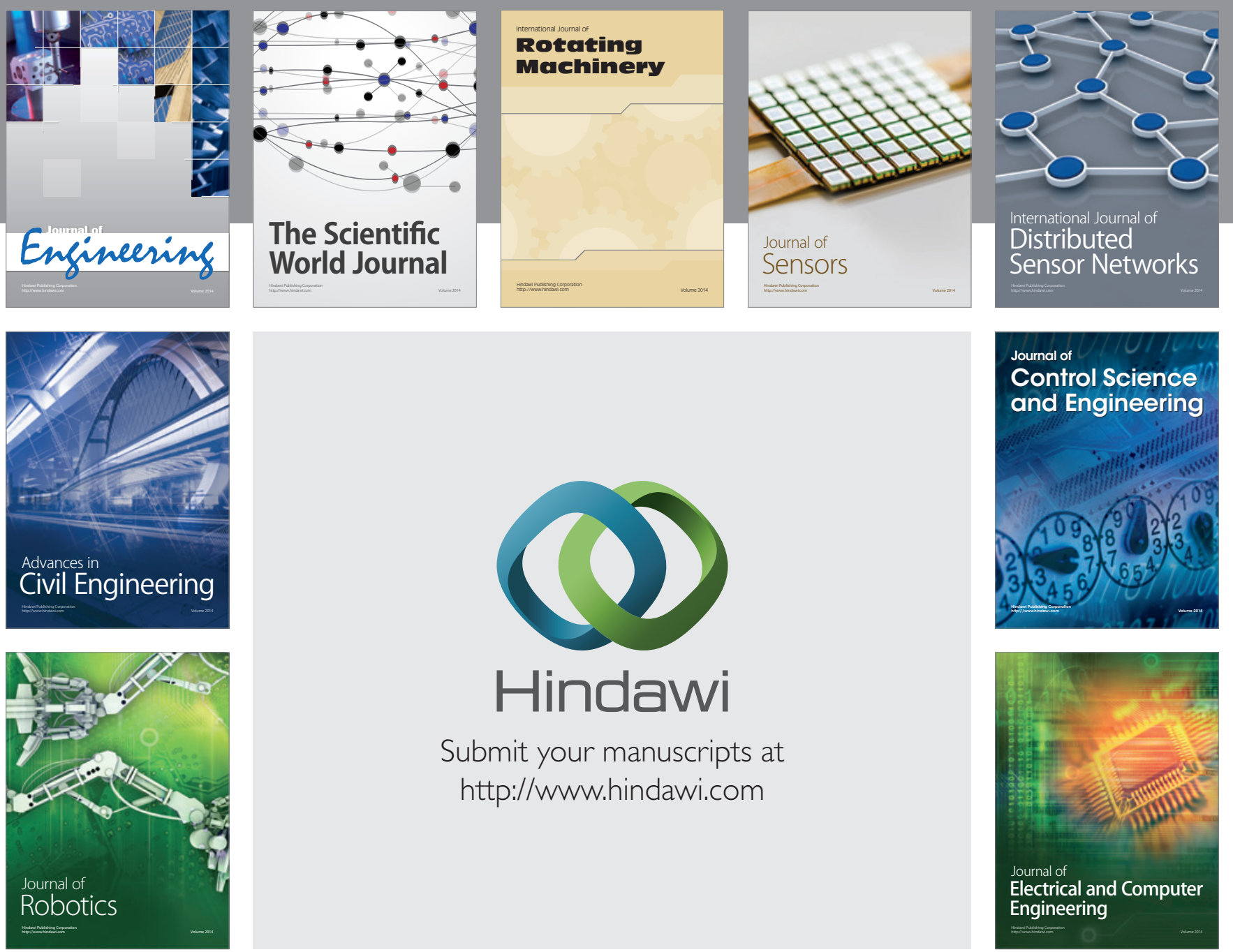

Submit your manuscripts at

http://www.hindawi.com
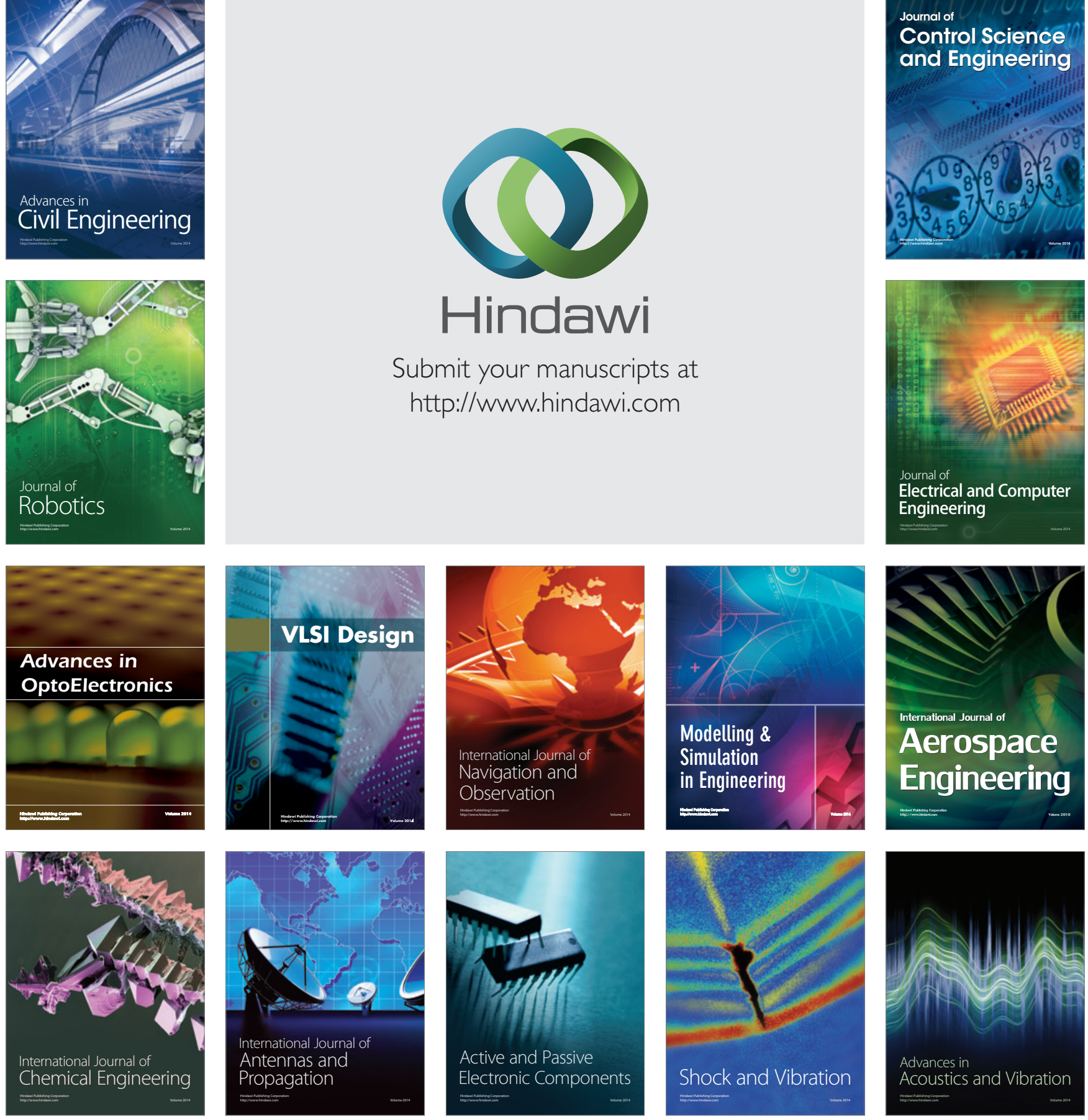